\title{
Prevalence and acquisition rate of methicillin resistant Staphylococcus aureus (MRSA) in internal medicine wards at the University Hospital of Geneva (HUG)
}

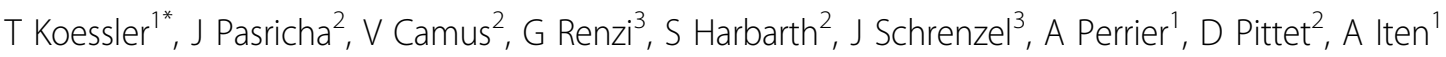 \\ From International Conference on Prevention \& Infection Control (ICPIC 2011) \\ Geneva, Switzerland. 29 June - 2 July 2011
}

\section{Introduction / objectives}

We aimed to determine the prevalence and acquisition rate of MRSA among patients admitted to internal medicine ward at HUG.

\section{Methods}

Patients consecutively admitted to 13 medical wards from March-June 2010 were screened for MRSA using pooled axilla and groin swabs within $48 \mathrm{~h}$ of admission and $36 \mathrm{~h}$ of discharge.

\section{Results}

Among 1967 patients, swabs were collected in 1740 $(88 \%)$ at admission and $712(36 \%)$ at discharge; with 687 (35\%) having both. Mean age was 64 years; $58 \%$ were male. $4.8 \%(84 / 1740)$ of patients were MRSA positive on admission, of which $79 \%$-for whom data were available (43/54) - had been MRSA positive on screening in the previous 6 months. Of patients who were not MRSA positive at admission, 5.8\% (29/496) had previous carriage. MRSA carriage at admission was associated with age $(\mathrm{p}=0.0016$, Wilcoxon rank sum test) and a positive MRSA swab in the previous 6 months $\left(\chi^{2} 233\right.$, $\mathrm{p}<0.0001) .3 .8 \%(27 / 710)$ of patients acquired MRSA during hospital stay - having a positive MRSA swab on discharge but not on admission. By Wilcoxon rank sum test, MRSA acquisition was associated with length of hospital stay $(\mathrm{p}=0.0009)$ and age $(\mathrm{p}=0.0087)$. No association was found between MRSA carriage or acquisition and sex, provenance, antibiotic use, requirement of intensive or high dependency care and type of medical ward.

\section{Conclusion}

$4.8 \%$ of patients admitted to general medical wards at our hospital were MRSA positive, the majority of these patients had previous MRSA carriage. 3.8\% of patients acquired MRSA during hospital stay.

\section{Disclosure of interest}

None declared.

\section{Author details}

${ }^{1}$ Department of Internal Medicine, University Hospital of Geneva, Geneva, Switzerland. '2Department of Infection Control, Geneva, Switzerland. ${ }^{3}$ Central Laboratory of Bacteriology, University Hospital of Geneva, Geneva,

Switzerland

Published: 29 June 2011

doi:10.1186/1753-6561-5-S6-P7

Cite this article as: Koessler et al:: Prevalence and acquisition rate of methicillin resistant Staphylococcus aureus (MRSA) in internal medicine wards at the University Hospital of Geneva (HUG). BMC Proceedings 2011 5(Suppl 6):P7. 